\title{
Miniaturized System with a Facile Isothermal Amplification Microfluidic Chip for Rapid Detection of Zika Viruses
}

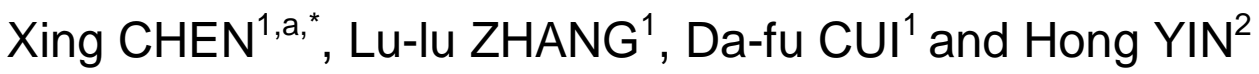 \\ ${ }^{1}$ State Key Laboratory of Transducer Tech, Institute of Electronics, Chinese Academy \\ of Sciences, Beijing, 100190, CHINA \\ ${ }^{2}$ Chinese Academy of Inspection and Quarantine, Beijing, 100123, CHINA \\ axchen@mail.ie.ac.cn \\ ${ }^{*}$ Corresponding author
}

Keywords: Microfluidic chips, Loop-mediated is other mal amplification (LAMP), Formatting, Miniaturized System.

\begin{abstract}
A real-time fluorescence detection biomedical miniaturized system with a disposable microfluidic chip was developed for loop-mediated isothermal amplification (LAMP) reactions. The miniaturized system was developed consisting of a mini heating plate, a temperature sensor, a temperature controller and an adjustable mechanical stage. Based on experimental result, the temperature of the heating plate is evenly distributed, and the error of the temperature is less than $1.3 \%$. The microfluidic chip was designed and fabricated by MEMS technology and thin-casting method. The volume of the LAMP reaction was changed from $25 \mu \mathrm{L}$ to $2 \mu \mathrm{L}$. The volume of the LAMP reaction was optimized. The nucleic acid of Zika viruses was amplified and detected in real-time mode with our miniatureized system by using above microfluidic chip. From the experiment results, the detection range was from 100 copies to $10^{6}$ copies. This biomedical system has the potential for point-of-care diagnostics (POCT) with the many advantages, such as not high cost, short analysis and detection time, not much reagent and sample consumption and so on.
\end{abstract}

\section{Introduction}

Recently isothermal amplification methods, not requiring any thermal cycling, have been reported and application [1,2]. When compared to Polymerase chain reaction (PCR) methods, loop-mediated isothermal amplification (LAMP) method is a novel gene amplification method under isothermal conditions $\left(60-65^{\circ} \mathrm{C}\right)$ without the need to accurately toggle the reaction mixture between different temperatures. With the development of microfluidics technology, many efforts have been focused on lab-on-a-chip or micrototal analysis systems. Lab-on-a-chip or micrototal analysis systems have some advantages like high throughput, short analysis time, small volumes and high sensitivities [3, 4], which can be capable of ready measurement of disease biomarkers in physiological fluids $[5,6]$.

Combination of LAMP and microfluidic technology will miniaturize the LAMP detection system and facilitate the realization of point-of-care (POC) pathogen detection. Uddin et. al. [7] reported a portable automatic end-point detection system for LAMP reactions on Microfluidic Compact Disk Platform. This system allows a rapid and automatic endpoint detection which could lead to the development of a point-of-care diagnosis device for foodborne pathogens detection in a resource-limited environment.Fang et. al. [8] reported a LAMP integrated microfluidic chip with optical fibers for detection of pathogens using absorbance detection, which was an octopus-like 
multiplex microfluidic loop-mediated isothermal amplification assay. However the fabrication process was complicated.

In this study, we develop a miniaturized system with a facile isothermal amplification microfluidic chip for rapid detection of Zika viruses (ZIKV). A dramatic increasing ZIKV infection in the Americas was reported in 2015. The World Health Organization (WHO) called for fast-tracked development of Zika virus diagnostics [9]. Confronting with the Zika virus epidemics, the development of rapid, scalable diagnostic devices and method is needed. Thus we develop a biomedical system with a microfluidic chip for real-time fluorescence detection of various viruses, which can be carried out in multiple point-of-care (POC) molecular diagnostic applications. ZIKV-LAMP reaction on our microfluidic chip with high sensitivity, specificity, and rapidity, which potentially enable LAMP assays to be highly portable for on-site analysis.

\section{Experimental Section}

\section{Materials and Reagents}

Sylgard 184 (PDMS) was purchased from Dow Corning (Midland, MI, USA). Liquid paraffin and low temperature paraffin were purchased from Sinopharm Chemical Reagent Beijing Co., Ltd. DNA Amplification Kit and Fluorescent Detection Reagent was obtained from Eiken China Co., Ltd.

According to the bioinformatic analysis, a conservative fragment of the ZIKV Envelop gene were synthesized and inserted in a pUC plasmid to establish the amplification target. Among the fragment, six primers of LAMP assay were designed according to the gene sequences of the reference virus using a PrimerExplorer V4 program. Details of the final primers are shown in Supplementary Table 1. All the primers were synthesized by Sunbiotech (Beijing, China).

\section{Microchip Fabrication}

Microchips were designed and fabricated with several microcells by using PDMS. Silicon positive moulds were prepared by MEMS technologies. After the silicon positive moulds were accomplished, PDMS layers with different structures were fabricated by using the thin-casting method. After oxygen plasma treatment, the glass and the PDMS layer were aligned and bonded together. The microfluidic chips were finished and ready to be used, shown in Fig 1.

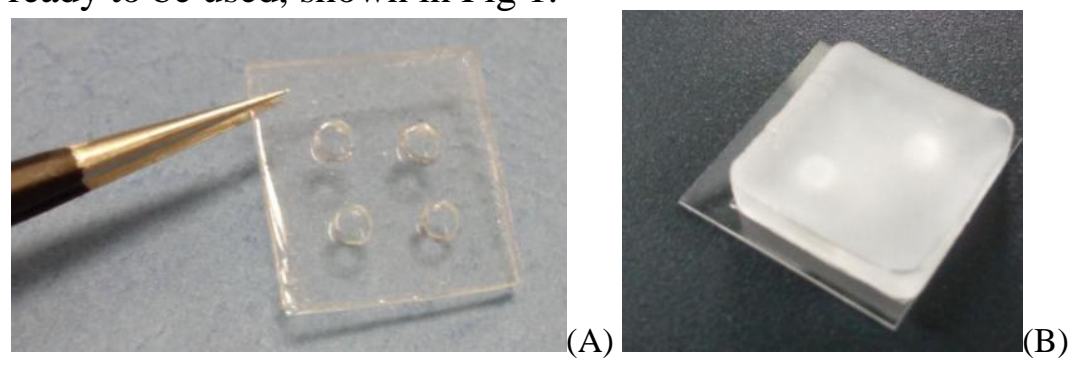

Figure 1. Photograph of the microfluidic chip with four microcells for LAMP reaction and with the low temperature paraffin as a cover.

\section{ZIKV-LAMP Reaction}

Routine LAMP assay was carried out with a Isothermal Master Mix (Optigene, the United Kingdom) in a final volume of 25 ul containing $0.125 \mathrm{nM}$ each of the outer primers F3 and B3, $1.0 \mathrm{nM}$ each of the primers FIP and BIP, and $0.5 \mathrm{nM}$ each of the 
primers LF and LB. The reaction mixture containing distilled water was used as negative controls.

\section{Miniaturized System}

The miniaturized system was developed consisting of a mini heating plate, a $\mathrm{Pt}$ temperature sensor, a PID temperature controller, a adjustable mechanical stage and a portable confocal fluorescent detector (ESE LOG ESML 10-MB-3007, Germany) shown in Fig. 2. This system with a microfluidic chip was used to implement the ZIKV-LAMP reaction and the real-time fluorescent detection of the amplification products. The results of LAMP procedure was output from the computer.

\section{Results and Discussion}

\section{Miniaturized System Setup}

The schematic diagram of the miniaturized system is shown in Fig 1. The real-time fluorescence detection was implemented by using the detection mode of single measurement for one hour. The interval between measurements was set at $1 \mathrm{~s} \sim 60 \mathrm{~s}$.
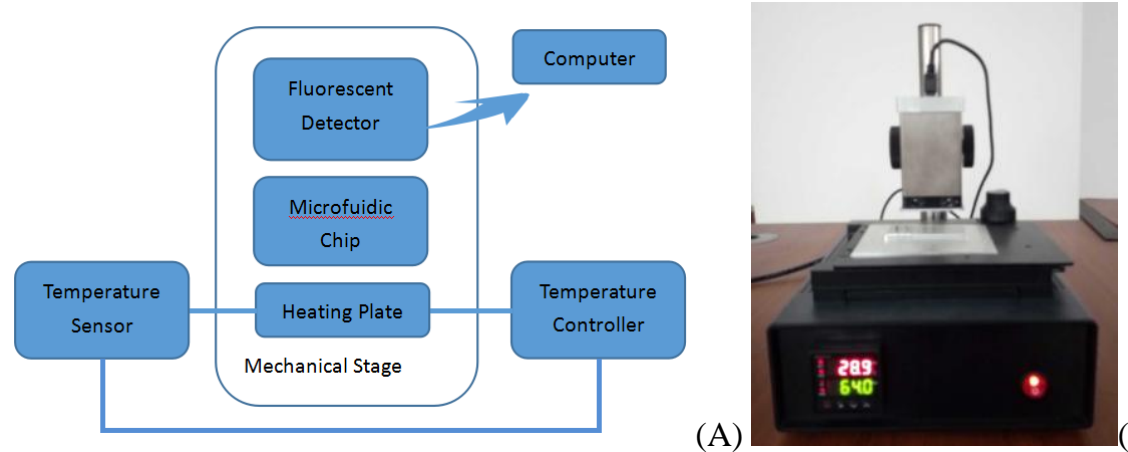

(A)

Figure 2. Schematic (A) and photograph (B) of the miniaturized system for real-time detection of ZIKV-LAMP reaction on a microfluidic chip.

Table 1 Temperature distribution at different locations at the rectangular heating plate.

\begin{tabular}{|c|c|c|c|c|}
\hline Test Position & Test 1 $\left({ }^{\mathbf{0}} \mathbf{C}\right)$ & Test 2 $\left({ }^{\mathbf{0}} \mathbf{C}\right)$ & Test 3 $\left({ }^{\mathbf{0}} \mathbf{C}\right)$ & Average Temperature $\left({ }^{\mathbf{0}} \mathbf{C}\right)$ \\
\hline $\mathbf{A}$ & 62.3 & 62.9 & 63.8 & 63 \\
\hline $\mathbf{B}$ & 62.8 & 61.8 & 63.9 & 63.8 \\
\hline $\mathbf{C}$ & 63.2 & 63 & 63.3 & 62.92 \\
\hline $\mathbf{D} *$ & 62.6 & 62.8 & 63.4 & 62.7 \\
\hline $\mathbf{E}$ & 62.7 & 62.8 & 62.5 & 62.3 \\
\hline $\mathbf{F}$ & 62.1 & 61.4 & 63.3 & 61.5 \\
\hline
\end{tabular}

*D represents the center position of the rectangular heating plate. The other six points, which are three rows and two columns, evenly distributes at the rectangular heating plate.

The temperature provided by the heating plate of the miniaturized system was carefully studied and the temperature was measured at different locations. The test results are shown in the table 1 . The size of the rectangular heating plate is $30 \mathrm{~mm} \times 70 \mathrm{~mm}$. Based on experimental result, the error of the temperature is less than $1.3 \%$ at different locations of the rectangular heating plate. The temperature difference varies from 0.1 to 0.8 degree Celsius. The temperature of the heating plate is evenly distributed, which can be used for heating three microfluidic chips with the size of $20 \mathrm{~mm} \times 20 \mathrm{~mm}$. 


\section{Optimization of Experimental Conditions}

Miniaturized system with a facile microfluidic Chip has been developed for the rapid LAMP reactions and the real-time fluorescence detection. LAMP reaction mixture was prepared in a centrifuge tube and then dropped in the microcell of the microfluidic chip. As shown in Fig 1, the size of the microfluidic chips is $20 \mathrm{~mm} \times 20 \mathrm{~mm}$ and the diameter of the microcells is about $3 \mathrm{~mm}$ and the depth is $2.5 \mathrm{~mm}$. Therefore the maximum volume of the microcells is about $17.7 \mu \mathrm{L} .10 \mu \mathrm{L}, 5 \mu \mathrm{L}$ and $2 \mu \mathrm{L}$ of LAMP reaction mixture were introduced into microfluidic chips, respectively, and then several microliters of liquid paraffin and low temperature paraffin were added to avoid evaporation during heating process. The amplification reactions in microchips were performed at $60{ }^{\circ} \mathrm{C}-65^{\circ} \mathrm{C}$ in our miniaturized system for $1 \mathrm{~h}$ (See Fig 2).

The microchip with the LAMP reaction mixture was placed on the mechanical stage, and the $x-y$ axis of the moving frame in the mechanical stage was adjusted to ensure that the fluorescence detector was aligned with the microcell. Then $\mathrm{z}$ axis of the moving frame in the mechanical stage was adjusted to ensure to obtain moderate fluorescence intensity. After determining the optimal position, the microchip was removed. The temperature switch of the heating plate was open and the temperature of heating plate was raise to the reaction temperature by using the temperature sensor and controller. After the temperature is stable, the chip was re-placed on the mechanical stage, while the fluorescence detector was open to start real-time testing. The experimental results are shown in Fig 3A. From the experimental results, dynamic curve of $2 \mu$ LAMP reaction is similar with that of the negative control, while dynamic curves of $5 \mu \mathrm{l}$ and 10 $\mu 1$ LAMP reaction is obviously different from the negative control. $10 \mu \mathrm{l}$ of the LAMP reaction volume has been used for the following experiments because of high FI.
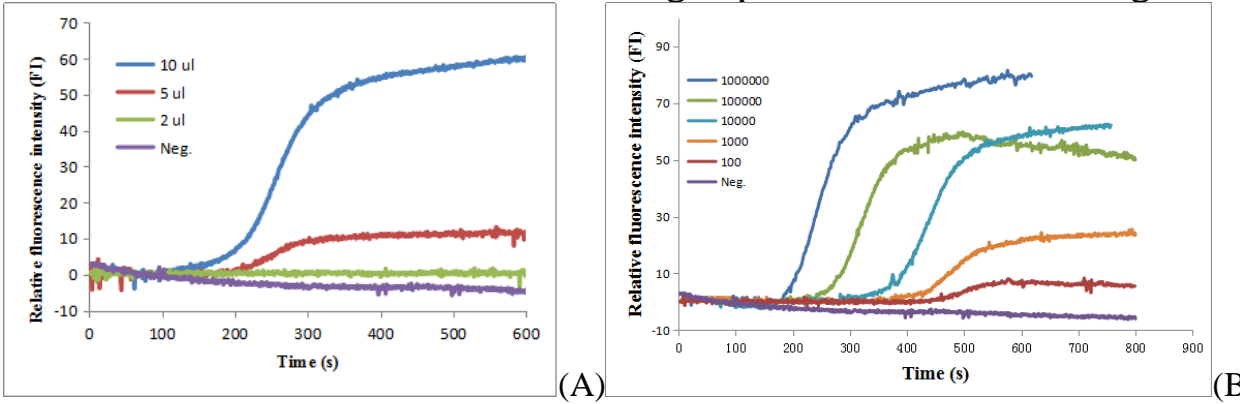

Fig 3. Dynamic curves of the real-time fluorescent detections of the amplification products of ZIKV-LAMP reactions on microfluidic chips by using our miniaturized system. Curves " $10 \mu \mathrm{l}$ ", " $5 \mu \mathrm{l}$ " and " $2 \mu \mathrm{l}$ " in (A) represent the optimization results with the volume of $10 \mu \mathrm{l}, 5 \mu \mathrm{l}$ and $2 \mu \mathrm{l}$, respectively. Curves "1000000" - "100" in (B) represent the optimization results of

1000000 copies - 100 copies in each ZIKV-LAMP reaction, respectively. Curve "Neg." represents the negative control.

\section{Real-time Fluorescence Detection of ZIKV}

The amplification products of LAMP reactions in microfluidic chips were directly real-time detected in our miniaturized system, which was also used to assess the method of the real-time fluorescence detection and our miniaturized system. Utilizing the above optimized condition, ZIKV-LAMP reactions were detected with serial ten-fold standard plasmids on the disposable microchips.

The dynamic cures of the real-time fluorescence detection of the ZIKV-LAMP reaction and the negative control on microfluidic chips by using our miniaturized system are shown in Fig. 3B. From Fig 3B, we found the difference between the positive ZIKV-LAMP reaction and the negative control is very various, which indicates that our real-time fluorescence detection miniaturized system with a microfluidic chip is successfully used for LAMP reaction. 
Also from the amplification results with serial ten-fold standard plasmids on the disposable microchips, the peak time is increased with the increasing template concentration of standard plasmids. Moreover dynamic curves of the real-time fluorescent detections of the $10^{5}-10^{3}$ template plasmid copies expressesd a dose-dependent positive amplification signals, similar with the detection results by Genie II instrument (shown in Supplementary Fig. 1). But the detection limit is lower than Genie II instrument, as the amplification signals can hardly be observed when the amplification templates are lower than $10^{2}$ copies/ reaction.

\section{Summary}

A miniaturized system with a disposable microfluidic chip was developed for ZIKV-LAMP reaction and real-time detection. The function of the system miniaturized has been verified by the LAMP experiments, which can provide the stable temperature. The error of the temperature was less than $1.3 \%$. Tthe volume of the reaction system is reduced from 25 microliters to 5 microliters because of using microfluidic chips. It is successfully used for IKV-LAMP reaction and real-time detection. The detection range was from 100 to 106 copies/reaction. This miniaturized system would be possible for on-site, low-cost POCT.

\section{Acknowledgement}

This research was financially supported by the National Science Foundation of China (61372055, 31571007, 61571420 and 8137171) and the National Key Scientific Instrument and Equipment Development Project (2012YQ090197).

\section{Supplementary Results}

Supplementary Table 1. Details of primers used for ZIKV-LAMP assay.

\begin{tabular}{lll}
\hline Primer name & $\begin{array}{l}\text { Genome } \\
\text { position }\end{array}$ & \multicolumn{1}{c}{ Sequence (5'-3') } \\
\hline F3 & $1495-1512$ & AAGCACTGGCTGGTTCAC \\
B3 & $1674-1691$ & TCCAGAGCTCCAGCAAGG \\
FIP(F1c+F2 & $(1555-1574)$ & GTGGAGTTCCGGTGTCTGCCAAGGAGTGGTTCCACGA \\
) & - & CAT \\
BIP(B1c+B & $(1513-1533)$ & \\
2) & - & AGAGTTCAAGGACGCACATGCCTGCTCCTTCTTGACTC \\
& $(1644-1663)$ & \\
LF & $(1534-1553)$ & CAGCGTGCCAAGGTAATGGA \\
LB & $(1622-1641)$ & AAAAGGCAAACTGTCGTGGT \\
\hline
\end{tabular}




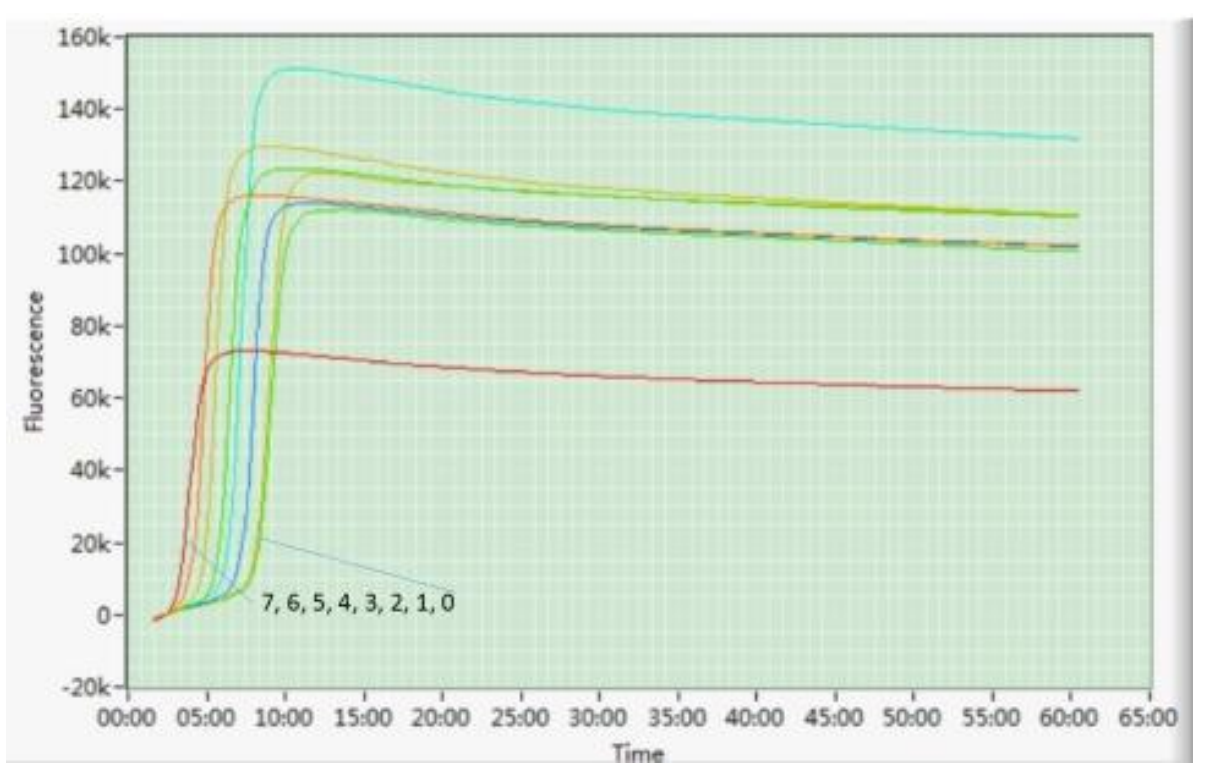

Supplementary Fig 1. Real time fluorescent LAMP assays of serial ten-fold standard plasmids. Digits of 7-0 indicate $10^{7}$ to $10^{\circ}$ copies/reaction as the reaction templates. $25 \mu$ L LAMP reaction mixture was added in centrifugal tubes. The amplification reactions in centrifugal tubes were carried out for 60 min using a real-time fluorescent Genie II instrument (Optigene, the United Kingdom) at $60^{\circ} \mathrm{C}-65^{\circ} \mathrm{C}$, which was proved that the ZIKV-LAMP reaction can proceed normally.

\section{References}

[1] T. Notomi, H. Okayama, H. Masubuchi, Loop-mediated isothermal amplification of DNA. Nucleic Acids Research, 28 (2000) e63.

[2] K. A. Curtis, D. L. Rudolph and S, M. Owen, Rapid detection of HIV-1 by reverse-transcription, loop-mediated isothermal amplification (RT-LAMP), Journal of Virological Methods, 151(2008)264-270.

[3] G. M. Whitesides, The origins and the future of microfluidics, Nature, 442 (2006) 368-373.

[4] A. Arora, G. Simone, G.B. Salieb-Beugelaar, Latest developments in micro total analysis systems, Analytical Chemistry 82 (2010) 4830-4847.

[5] B. S. Ferguson, S. F. Buchsbaum, T. T. Wu, Genetic analysis of H1N1 influenza virus from throat swab samples in a microfluidic system for point-of-care diagnostics, Journal of the American Chemical Society, 133(2011)9129-9135.

[6] S. Lingam, M. Beta, D. Dendukuri, A focus on microfluidics and nanotechnology approaches for the ultra sensitive detection of microRNA, Microrna, 3(2014)18-28

[7] S. M. Uddin, F. Ibrahim, A. A. Sayad, A Portable Automatic Endpoint Detection System for Amplicons of Loop Mediated Isothermal Amplification on Microfluidic Compact Disk Platform, Sensors, 15(2015)5376-5389.

[8] X. Fang, Y. Liu, J. Kong and X. Jiang, Loop-Mediated Isothermal Amplification Integrated on Microfluidic Chips for Point-of-Care Quantitative Detection of Pathogens, Analytical Chemistry, 82(2010)3002-6.

[9] K. Pardee, A. A. Green, M. K. Takahashi, et al. Rapid, Low-Cost Detection of Zika Virus Using Programmable Biomolecular Components, Cell, 165(2016)1255-66. 